\title{
La distribución geográfica de la tángara azul-gris (Thraupis episcopus) en hábitats modificados antropogénicamente en México
}

\section{The geographical distribution of the Blue-gray Tanager (Thraupis episcopus) through anthropogenically modified habitats in Mexico}

\author{
Erick Rubén Rodríguez-Ruíz ${ }^{*}$, Héctor Arturo Garza-Torres², César A. Ríos-Muñoz y Adolfo G. \\ Navarro-Sigüenza ${ }^{3}$ \\ ${ }^{1}$ Instituto Tecnológico de Ciudad Victoria. Blvd. Emilio Portes Gil 1301 Pte., 87010 Ciudad Victoria, Tamaulipas, México. \\ ${ }_{2}^{2}$ Instituto de Ecología Aplicada, Universidad Autónoma de Tamaulipas. Av. División del Golfo 356, Col. Libertad, 87019 Ciudad Victoria, Tamaulipas, \\ México. \\ ${ }^{3}$ Museo de Zoología “Alfonso L. Herrera”, Facultad de Ciencias, Universidad Nacional Autónoma de México. Apartado postal 70-399, 04510 México \\ D. F. México. \\ *Correspondencia: erick_burrin@yahoo.com
}

\begin{abstract}
Resumen. Mediante trabajo de campo, la revisión de bases de datos biológicos e información de la distribución geográfica histórica y actual de Thraupis episcopus se establecieron nuevos registros de anidación en el noreste de México y su ampliación hacia hábitats modificados por actividades humanas en la península de Yucatán y el noreste de México. Además, se evaluó si existía preferencia de la especie por hábitats conservados vs. modificados, por medio de un análisis de escenarios de cambio de uso de suelo de 3 décadas diferentes (1970, 1980 y 1990), obteniendo porcentajes de presencia. Se encontró que no existe una diferencia significativa entre preferencias de hábitat a través del tiempo.

Palabras clave: tángara azul-gris, áreas urbanas, registros de anidación, cambio de uso de suelo, expansión geográfica, este de México.

Abstract. We analyzed the historical and current distributional range data of the Blue-gray Tanager, and reported new nesting records in northeastern Mexico. We also assessed the range extension of this species into human-altered habitats in the Yucatán Peninsula and northeastern Mexico, based both on records from fieldwork and biological databases. We identified habitat preference (conserved vs. human altered) using land use change scenarios from 3 decades (1970, 1980, and 1990) and percentages of occurrence in a multitemporal approach, finding no significant differences in habitat use through time.
\end{abstract}

Key words: Blue-gray Tanager, urban areas, nesting records, land use change, geographic expansion, eastern Mexico.

\section{Introducción}

Desde principios del siglo XX, las modificaciones antropogénicas de las áreas naturales de México han generado un gran trastorno ambiental para la flora y fauna, lo cual se refleja en cambios en sus poblaciones y en reducciones o ampliaciones de su distribución (Escalante et al., 1998; Ceballos y Márquez-Valdelamar, 2000). La parte más afectada se concentra en los bosques tropicales perennifolios y caducifolios de tierras bajas, con una modificación hasta del 50\% para el año 2000 (Trejo y Dirzo, 2000; Velázquez et al., 2002), reflejando una gran extensión que ha sido modificada en la planicie costera

Recibido: 06 enero 2010; aceptado: 27 enero 2011 del golfo y la costa de Chiapas en el sur de México, especialmente para desarrollar actividades agropecuarias (Peterson et al., 2006). Para algunos taxones, la modificación del hábitat por factores antropogénicos en áreas naturales afecta de forma significativa varios procesos biológicos (Challenger y Caballero, 1998; CONABIO, 2006).

$\mathrm{Se}$ conoce que algunas especies de aves aparentemente se benefician de esos hábitats modificados antropogénicamente, incluso algunas son consideradas como bioindicadoras de perturbación, sean residentes (e. g., Quiscalus mexicanus, Turdus grayi, Pitangus sulphuratus, Columbina inca, Molothrus aeneus, Zenaida asiatica y Z. macroura), migratorias (e. g., Falco peregrinus, Mniotilta varia, Tyrannus forficatus, Wilsonia pusilla) o exóticas (e. g., Passer domesticus, Streptotelia 
decaocto, Columbia livia, Myiopsitta monachus; Álvarez-Romero et al., 2008). Esas especies utilizan estos hábitats por la fácil disposición de alimento o por el bajo número de depredadores naturales, proporcionando sitios de refugio, percha o anidación. Al aumentar su adecuación, estas especies pueden llegar a mantener poblaciones estables a lo largo del tiempo (MacGregor, 2005; Ramírez-Albores, 2008; Álvarez-Romero et al., 2008; MacGregor-Fors, 2008; MacGregor-Fors et al., 2008; Pablo, 2009).

Recientemente se han llevado a cabo estudios detallados en torno al papel que desempeñan las aves en hábitats modificados antropogénicamente, generando información acerca de los procesos de interacción de las especies asociadas a las modificaciones en su entorno. Un ejemplo es el producido por el cambio de uso de suelo. Pocos son los estudios realizados en México donde se analizan estos factores. Sánchez-Cordero y Martínez-Meyer (2000) y Sánchez-Cordero et al. (2005) hacen mención de la afectación y uso positivo de los hábitats modificados por el cambio de uso de suelo para algunos mamíferos. Para las aves, Peterson et al. (2006) sugirieron la aplicación de nuevos métodos en la identificación histórica del cambio de uso de suelo y modelos de nicho ecológico, que sirvan como herramienta geográfica para explorar las condiciones actuales para este grupo. Estos autores hacen énfasis en la pérdida acelerada de poblaciones de córvidos mexicanos debido a la modificación y pérdida de hábitats naturales, particularmente para especies estrechamente asociadas a ciertos tipos de ecosistemas. Ríos-Muñoz y NavarroSigüenza (2009) documentaron la pérdida del hábitat potencial en las últimas 3 décadas para los psitácidos mexicanos debido al cambio de uso de suelo y por tanto, la reducción masiva en su área de distribución.

En este escrito se presenta información sobre la distribución actual en México de la tángara azul-gris (Thraupis episcopus), una especie neotropical, residente y común, que se distribuye desde el este de México hasta el centro de Brasil, norte de Bolivia y este de Perú (Clements, 1991; Dickinson, 2003). Este traúpido tiene preferencia por áreas abiertas y semiabiertas, plantaciones, jardines en las afueras de las ciudades, parques, plazas, bordes de bosques húmedos, y bosques secundarios (Peterson y Chalif, 1989; Howell y Webb, 1995; Silva et al., 1996). Se encuentra entre los 0 y los $1500 \mathrm{~m}$ snm (AOU, 1998). Prospera en asentamientos humanos, vive en pareja o en grupos casi todo el año, además de ser muy activo y ruidoso (Isler e Isler, 1987). En México, su distribución incluye los estados del sureste (Howell y Webb, 1995; AOU, 1998) contando recientemente con registros en Nuevo León (Monroy y Grosselet, 2008). En este estudio se documenta el efecto de cambio de uso de suelo en la distribución de T. episcopus a lo largo del territorio mexicano, y se presenta información detallada acerca de nuevos sitios de anidación en el noreste de México.

\section{Materiales y métodos}

Con el fin de obtener todos los registros conocidos para la especie en México, se consultaron diferentes fuentes bibliográficas (Lowery y Newman, 1951; Davis, 1952; Boyd, 2001; Navarro et al., 2003; Monroy y Grosselet, 2008), la Red Mundial de Información sobre Biodiversidad (http://www.conabio.gob.mx/remib/ doctos/ remibnodosdb.html) y la Avian Knowledge Network (AKN, 2009; http://www.avianknowledge.net). Estos datos se complementaron con registros originales recientes que provienen de observaciones personales y de monitoreos de aves, realizados bajo el protocolo de sobrevivencia invernal (MoSI) en la Reserva de la Biosfera El Cielo, de 1997 a 2009, y de diferentes inventarios realizados en Tamaulipas, San Luis Potosí y la parte norte de Veracruz. A partir de estos registros, se construyó una base de datos que fue visualizada en un sistema de información geográfica (ArcView 3.2., ESRI, 1999).

Para evaluar y comparar los registros puntuales de la especie con respecto al cambio de uso de suelo, se utilizaron mapas digitales de 3 escenarios de uso de suelo y vegetación, basados en el mapa de vegetación potencial de Rzedowski (1978, 1990), los cuales se tomaron como una hipótesis de los tipos de vegetación naturales para México sin considerar el impacto de las actividades humanas (Peterson et al., 2006; Ríos-Muñoz y Navarro-Sigüenza, 2009).

Para el manejo de estos escenarios, se establecieron criterios que uniformaran la información y poder hacer las comparaciones entre las distintas fechas, con base en lo propuesto por Peterson et al. (2006) y Ríos-Muñoz y Navarro-Sigüenza (2009), considerando como zonas modificadas las áreas sin vegetación nativa aparente, acahuales, pastizales inducidos, zonas urbanas, agrícolas y pecuarias (SEMARNAT/ IG-UNAM, 2001). Lo anterior fue necesario debido a que las coberturas de uso de suelo y vegetación provienen de 2 fuentes, una con información de fotografía aérea (1973 a 1979; Peterson et al., 2006) y otra de imágenes satelitales LandSat (1996 y 2000; SEMARNAT/ IG-UNAM, 2002; Velázquez et al., 2002; Peterson et al., 2006).

Para establecer el grado de asociación de estas especies con el hábitat, se contabilizaron las localidades donde la especie fue registrada (datos puntuales) asociándolas directamente con las coberturas de cambio de uso de suelo ya uniformadas, considerando los hábitats conservados y 
modificados antropogénicamente. Mediante esta asociación se pudo obtener el número de localidades directamente vinculadas con los tipos de hábitat clasificados. Para la realización de estas comparaciones fue preciso que tanto los datos puntuales y los escenarios de cambio de uso de suelo tuvieran congruencia temporal. Una vez obtenida la sumatoria de las localidades en cada escenario, se analizó de manera multitemporal, comparando los porcentajes totales de localidades por tipo de hábitat en los 3 escenarios. La visualización geográfica de los datos, permitió identificar modificaciones espacio-temporales de la especie y su correspondencia con los tipos de hábitat.

Con la finalidad de probar si la especie utilizó los 2 tipos de hábitat de una forma diferencial a través del tiempo, se realizó una prueba de paralelismo o de homogeneidad de pendientes con los valores de número de registros por hábitat en cada década (Zar, 1999). Esta prueba permite evaluar diferencias significativas entre las pendientes producto de una serie de regresiones lineales (una por década) del número de registros de la especie asociados a cada tipo de hábitat. Para ello se utilizó el programa Statistica C) (StatSoft, 1998).

\section{Resultados}

Para T. episcopus se obtuvieron 722 registros primarios de presencia (especie-localidad-fecha) en México, distribuidos en 323 localidades únicas, que abarcan un intervalo temporal de los años 1866 a 2009. Vale la pena señalar que tan sólo para el noreste de México existen 47 registros puntuales recientes (posteriores al año 1999), además de los de anidación, distribuidos en 20 localidades (Fig. 1).

Se crearon 3 mapas en los que se representa la distribución geográfica de la especie en México, cada uno representa un escenario basado en la congruencia temporal entre registros y coberturas (Fig. 1). Los porcentajes de registros puntuales por localidad, considerando los hábitats conservados y modificados, refleja un incremento en el número de registros de la especie a través de las últimas décadas (Cuadro 1). El número de localidades en sitios conservados y modificados antropogénicamente no parece ser equivalente, pues en el escenario de la década de 1980 no existen registros para las zonas conservadas. Sin embargo, puede observarse de manera general, que del total de los registros, comparando las 3 décadas, el número acumulado en zonas conservadas y modificadas no difiere a través del tiempo (análisis de paralelismo; $p=0.93$, g.l.= 2), indicando que no existen diferencias que sugieran la preferencia de la especie entre hábitats conservados y perturbados (Fig. 1).

Por medio del análisis geográfico de los datos históricos fue posible identificar que la concentración de registros previos a la década de 1970 corresponde a la parte central de la vertiente del golfo de México. A partir de 1970 empiezan a aparecer registros aislados en el noreste de México. Para la década de 1980 aumenta el número de registros en esta zona, sobre todo en áreas del este de San Luis Potosí. No obstante, sólo en la década de 1990 aparecen registros para la península de Yucatán y un aumento significativo para San Luis Potosí. Cabe señalar que en los registros recientes (2000 a 2009) se percibe un aumento considerable para todo el noreste de México y la península de Yucatán, incluso en los de anidación en áreas urbanas (Monroy y Grosselet, 2008). Estos registros recientes coincidieron principalmente en áreas modificadas por actividades humanas, pues la región de la vertiente del golfo de México presenta mayor aumento de degradación de hábitat dentro del área de distribución de la especie, sobre todo para el periodo 1970 a 2009 (Fig. 1).

Durante el trabajo de campo se obtuvieron 26 registros localizados en 9 localidades de los estados de San Luis Potosí (Cd. Valles) y Tamaulipas (Ciudad Victoria, Gómez Farías, Ojo de Agua, Azteca, El Mante, El Nacimiento, Tancol y Tampico), ubicados todos en áreas con modificaciones antropogénicas. En parte, la vegetación de estos sitios está representada por plantas exóticas, como ficus (Ficus benjamina), trompeta amarilla (Tecoma stans), jacaranda (Jacaranda mimosifolia), anacahuita (Cordia boissieri), cedro rojo (Cedrela odorata), fresno americano (Fraxinus americana), árbol de chaca (Bursera simaruba), almendro de la India (Terminalia catappa), ciprés común (Cupressus sempervirens), huamúchil (Pithecellobium dulce) y mirabobo (Melia azedarach), y en su mayoría

Cuadro 1. Número y porcentaje de registros puntuales de T. episcopus en las 3 décadas con coberturas de cambio de uso de suelo, en hábitats conservados vs. modificados antropogénicamente

\begin{tabular}{cccc}
\hline Décadas & $\begin{array}{c}\text { Localidades con hábitats } \\
\text { conservados }\end{array}$ & $\begin{array}{c}\text { Localidades con hábitats } \\
\text { modificados }\end{array}$ & Total de registros \\
\hline 1970 & $16(69.5 \%)$ & $7(30.4 \%)$ & 23 \\
1980 & $0(0 \%)$ & $13(100 \%)$ & 13 \\
1990 & $38(59.3 \%)$ & $26(40.6 \%)$ & 64 \\
Total & $54(54 \%)$ & $46(46 \%)$ & 100 \\
\hline
\end{tabular}



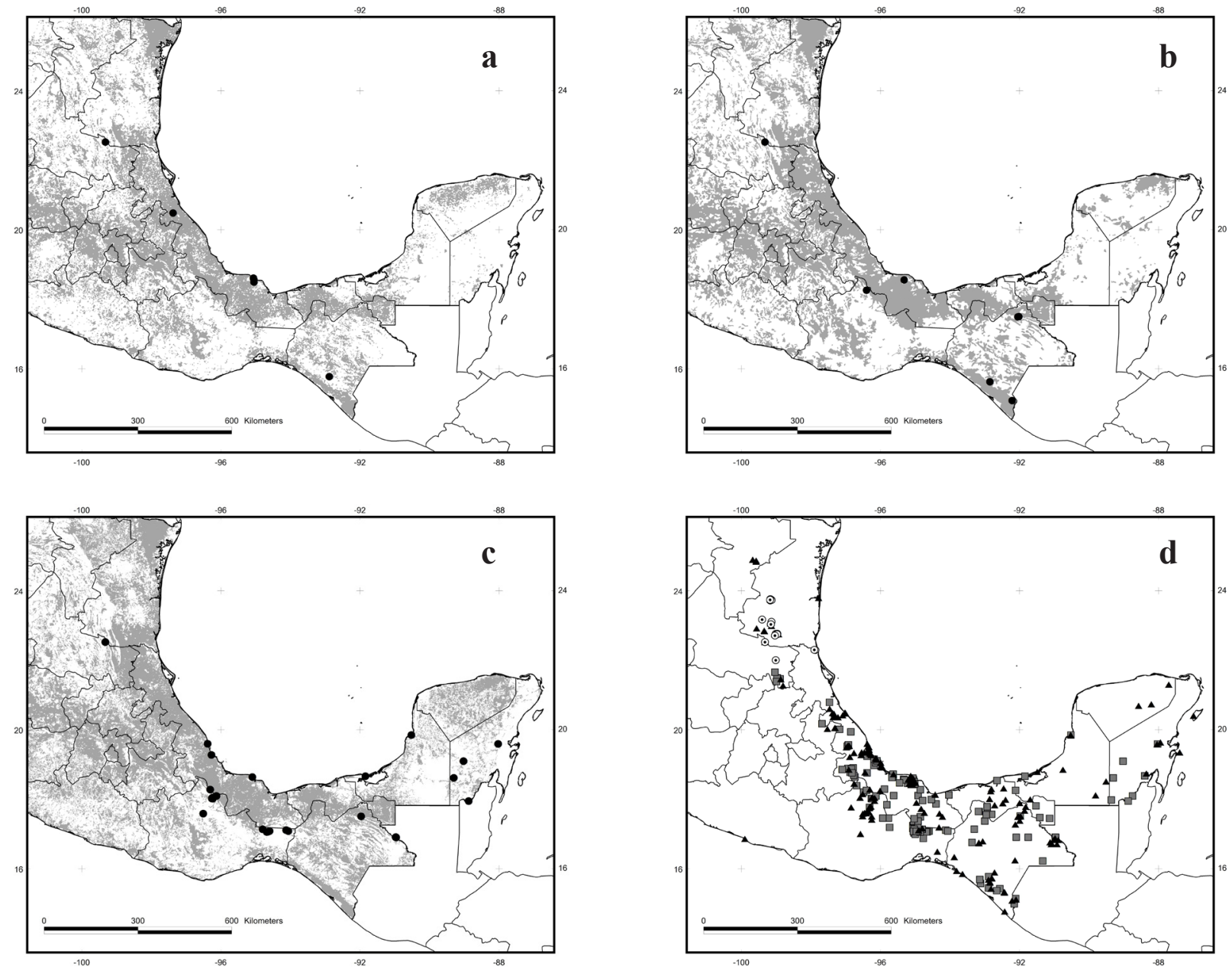

Figura 1. Mapas digitalizados con los escenarios de cambio de uso de suelo en 3 décadas. a) 1970; b) 1980; c) 1990. Las áreas modificadas antropogénicamente están representadas en gris y se sobreponen los registros puntuales de T. episcopus correspondientes a cada década, que se denotan con círculos negros (•); d) registros puntuales de T. episcopus para México: datos del Atlas de las aves de México (Navarro et al. 2003), cuadros grises ( $\square$ ); registros de la AKN (2009), triángulos negros ( $\bullet$ ); registros obtenidos en el trabajo de campo de este estudio, círculos $(\odot)$.

los registros fueron cerca de cuerpos de agua. Además, se pudo observar que la especie permanecía en grupos de 2 a 3 individuos. Se observó que los primeros registros de anidación fueron en Tamaulipas, para 2 sitios de áreas urbanas de El Mante; el primero en la colonia Miguel Alemán (22 $45^{\circ} 06^{\prime \prime} \mathrm{N} 098^{\circ} 58^{\prime} 00^{\prime \prime} \mathrm{O}$; $\left.86 \mathrm{~m} \mathrm{snm}\right)$ y el segundo en el fraccionamiento Linares $\left(22^{\circ} 45^{\prime} 17^{\prime \prime} \mathrm{N}\right.$ 099 00’38" O; 56 m snm).

\section{Discusión}

Thraupis episcopus es una de las especies de tráupidos para las que existe mayor información sobre su biología general (Isler e Isler, 1987; Skutch, 1989), incluyendo sus hábitos reproductivos (Foster, 1974; Haverschmidt, 1954;
Ingels, 1978; Gómez et al., 2000), alimenticios (Snow y Snow, 1971), patrones estacionales (Silva et al., 1996) y de distribución (AOU, 1998; Clements, 1991; Dickinson, 2003). Sin embargo, la aparición de registros que amplían su distribución a la península de Yucatán y en el noreste de México han sido documentados hace relativamente poco tiempo (Navarro et al., 2003; Monroy y Grosselet, 2008; AKN, 2009). Aunque existe la posibilidad de que los registros que amplían su distribución puedan deberse a ejemplares escapados accidentalmente de cautiverio (Instituto Nacional de Ecología, 1997; Monroy y Grosselet, 2008), no existe información detallada que pudiera corroborar su comercialización. Además, los registros de observaciones de AKN (2009) y los de este estudio no parecen ser aislados, ya que la visualización geográfica 
de la especie permitió identificar un patrón constante de expansión de su distribución hacia el noreste de México y en la península de Yucatán (Navarro et al., 2003; AKN, 2009; Fig. 1). Los registros puntuales de la especie en estas 2 regiones se obtuvieron en hábitats modificados antropogénicamente (SEMARNAT/ e IG-UNAM, 2001; Peterson et al., 2006). Este patrón está confirmado por los resultados de campo, ya que en sitios conservados de áreas como la sierra Madre Oriental, sierra de Tamaulipas, sierra de San Carlos, laguna Madre, los humedales del sur de Tamaulipas y norte de Veracruz, y el altiplano tamaulipeco no fue localizada la especie, a pesar de la búsqueda focal que se llevó a cabo.

Aunque no existen coberturas de cambio de uso de suelo que estén actualizadas y disponibles para los últimos 10 años, se ha podido observar que las localidades de registro posteriores al año 2000 corresponden en un $46 \%$ a sitios que ya estaban modificados desde finales de la década de 1990. En conjunto, los resultados obtenidos muestran que no existe asociación marcada con la condición del hábitat. Esto sugiere la capacidad de esta especie para explotar condiciones tanto conservadas como modificadas antropogénicamente (Cuadro 1; Silva et al., 1996; Monroy y Grosselet, 2008).

Hay que resaltar que en México aún son pocos los trabajos en los que se ha evaluado el efecto del cambio de uso de suelo sobre la diversidad y la distribución de las especies animales (Sánchez-Cordero y Martínez-Meyer, 2000; Sánchez-Cordero et al., 2005; Peterson et al., 2006; MacGregor-Fors et al., 2008; Ríos-Muñoz y NavarroSigüenza, 2009) y el hecho de que las coberturas utilizadas hayan sido generadas en diferentes tiempos y con distintos métodos (SEMARNAT/ IG-UNAM, 2001; Peterson et al., 2006), puede causar que su compatibilidad no sea total, a pesar de ser completas y precisas. Debido a esas limitantes, dichos estudios de cobertura sólo son adecuados para realizar análisis a escala nacional y para entender patrones generales. A escalas locales pueden presentarse problemas, dado que la información de uso de suelo no coincide en su totalidad, y por lo tanto puede presentar información que no se ajuste a la realidad (Ríos-Muñoz y NavarroSigüenza, 2009).

En los últimos años ha habido registros de aves neotropicales que amplían su distribución como respuesta a las actividades humanas (e.g., Turdus rufopalliatus, Martínez-Morales et al. 2010), hacia el noreste de México (e. g., Garza-Torres y Navarro, 2003; Garza-Torres et al., 2003; Garza-Torres, 2007; Monroy y Grosselet, 2008; Monroy y Sánchez, 2009; Brush, 2009) y en algunos casos hacia la península de Yucatán (Rojas-Soto y de Alba, 2002; Correa-Sandoval et al., 2006; Ibáñez-Hernández y ÁlvarezSolórzano, 2007), tal como sucede con T. episcopus. Sin embargo, en ambas regiones el impacto provocado por el cambio de uso de suelo, también ha causado reducción en las áreas de distribución y el tamaño de las poblaciones de otras especies de aves en niveles que van de moderados a altos.

La cotorra cabeza roja (Amazona viridigenalis) ha sufrido una reducción hasta del $58 \%$ en su área de distribución para el noreste de México, y para el loro yucateco (Amazona xantholora) existe una reducción del 25.91\% del hábitat potencial para el año 2000 en la península de Yucatán, causada por las modificaciones antropogénicas al hábitat (Ríos-Muñoz y NavarroSigüenza, 2009). Por lo tanto, la modificación de la vegetación nativa que para algunas especies pudiera ser una causa de pérdida de hábitat ha sido aprovechada por otras, expandiendo sus áreas de distribución geográfica.

Este cambio en las preferencias de hábitat y modificaciones en el entorno de $T$. episcopus puede estar afectando las interacciones entre los taxones asociados, resultando en una respuesta positiva o negativa dependiendo del taxón y de la interacción resultante (Sekercioglu et al., 2004; Sekercioglu, 2006). En México aún se desconocen los cambios en la composición, la estructura y el funcionamiento de la avifauna en hábitats modificados antropogénicamente.

Resulta en particular notorio el reemplazo de las comunidades naturales por zonas modificadas antropogénicamente, reduciendo las áreas naturales a sólo manchones entre mosaicos de áreas agropecuarias y urbanas, lo que resulta grave para muchas especies de aves (Peterson et al., 2006; Ríos-Muñoz y Navarro-Sigüenza, 2009). Las áreas modificadas por perturbaciones humanas pueden convertirse en corredores biológicos que conecten a otras áreas sin previo registro de las especies que los aprovechan (Sekercioglu et al., 2004; Sekercioglu, 2006). En ciertos casos se ha observado que algunas especies, como Geothlypis policephala, aprovechan las zonas modificadas para desplazarse entre manchones de hábitats conservados (McGregor-Fors et al., 2008, MartínezMorales et al., 2010).

Aunque T. episcopus se mueve entre parches de vegetación secundaria y pastizales inducidos en áreas del Amazonas (Silva et al., 1996), en general parece preferir hábitats con alteraciones antropogénicas (Peterson y Chalif, 1989; Howell y Webb, 1995; AOU, 1998). A pesar de que en los registros históricos existe una proporción equitativa en su preferencia por hábitats conservados contra modificados, en los últimos años la especie no se ha registrado en áreas conservadas -significativamente en México-, sino en zonas modificadas. Esto indica que T. episcopus, a diferencia de otras especies, ha logrado prosperar en estas áreas modificadas, convirtiéndolas en su hábitat de distribución actual. 


\section{Agradecimientos}

A J. Valencia-Herverth, R. Pérez-Castañeda y B. Ruiz-Ruiz por sus sugerencias y comentarios durante la realización de este trabajo. A las colecciones que proporcionaron información de ejemplares de Thraupis episcopus: American Museum of Natural History, Academy of Natural Sciences of Philadelphia, Bell Museum of Natural History, University of Minnesota, British Museum (Natural History), Zoologische Museum Alexander Koenig, Carnegie Museum of Natural History, Centro de Investigaciones de Quintana Roo (Ahora en ECOSUR Chetumal), Canadian Museum of Nature, Cornell University Museum of Vertebrates, Delaware Museum of Natural History, El Colegio de la Frontera Sur Chetumal, Field Museum of Natural History, Colección Nacional de Aves, Instituto de Biología, UNAM, Instituto de Historia Natural y Ecología de Chiapas, University of Kansas Natural History Museum, Los Angeles County Museum of Natural History, Natuurhistorische Musem Leiden, Louisiana State University Museum of Zoology, Museo de las Aves de México, Museo de la Biodiversidad Maya Universidad Autónoma de Campeche, Museum of Comparative Zoology Harvard University, Moore Laboratory of Zoology, Museum Nationale d'Histoire Naturelle Paris, Museo Regionali di Scienze Naturali Torino, Museo de Zoología, Facultad de Ciencias UNAM, Royal Ontario Museum, Sistema Nacional de Información sobre Biodiversidad (CONABIO), Southwestern College, University of Arizona, Florida Museum of Natural History, University of Michigan, Museum of Zoology, United States National Museum of Natural History, Burke Museum University of Washington, Western Foundation of Vertebrate Zoology, y Peabody Museum Yale University. Erick Rubén Rodríguez-Ruíz agradece en particular el apoyo económico al proyecto SEMARNAT-CONACYT C01-0265 para realizar la estancia académica en el Museo de Zoología de la Facultad de Ciencias de la UNAM.

\section{Literatura citada}

Álvarez-Romero, J. G., R. A. Medellín, A. Oliveras de Ita, H. Gómez de Silva y O. Sánchez. 2008. Animales exóticos en México: una amenaza para la biodiversidad. CONABIO/ Instituto de Ecología,UNAM/ SEMARNAT, México, D. F. $502 \mathrm{p}$.

AOU (American Ornithologists'Union). 1998. Check list of North American birds, séptima edición, American Ornithologists' Union, Washington, D. C. 829 p.

AKN (Avian Knowledge Network ). 2009. The Bird Reference Dataset. http://www.avianknowledge.net/; última consulta: 10.IX.2009.
Boyd, R. L. 2001. New distributional records of two tanagers in Mexico. Cotinga 15:58-59.

Brush, T. 2009. Range expansion and new breeding records of birds in Tamaulipas, Mexico. Southwestern Naturalist 54:9196.

Ceballos, G. y L. Márquez-Valdelamar. 2000. Las aves de México en peligro de extinción. CONABIO/ FCE/ Instituto de Ecología, UNAM, México, D. F. 430 p.

Challenger, A. y J. Caballero. 1998. Utilización y conservación de los ecosistemas terrestres de México: pasado presente y futuro. CONABIO/ Instituto de Biología, UNAM/ Agrupación Sierra Madre, México, D. F. 847 p.

Clements, J. F. 1991. Birds of the world a check list, cuarta edición. Ibis, Vista, California. 617 p.

CONABIO (Comisión Nacional para el Conocimiento y Uso de la Biodiversidad). 2006. Red Mundial de Información sobre Biodiversidad. http://www.conabio.gob.mx/remib/doctos/ remibnodosdb.html; última consulta: 10.IX.2009.

Correa-Sandoval, J., A. de Alba, M. Tuz, H. Bahena, E. May y S. I. Pérez. 2006. Xanthocephalus xanthocephalus nuevo registro para la parte continental de la península de Yucatán, México. Huitzil 7:18-19.

Davis, L. I. 1952. Winter bird census at Xilitla, San Luis Potosí, Mexico. Condor 54:345-355.

Dickinson, E. C. (ed.). 2003. The Howard and Moore, complete checklist of the birds of the world, tercera edición. Princeton University Press, New Jersey. 1039 p.

Escalante, P., A. G. Navarro S. y A. T. Peterson. 1998. Un análisis geográfico, ecológico e histórico de la diversidad de aves terrestres de México. In Diversidad biológica de México. Orígenes y distribución, T. P. Ramamoorthy, R. Bye, A. Lot y J. Fa. (eds.). Instituto de Biología, UNAM. México, D. F. 279-303 p.

ESRI, 1999. ArcView v. 3.2. Environmental Systems Research.

Foster, M. S. 1974. Rain, feeding behavior, and clutch size in tropical birds. Auk 91:722-726.

Garza-Torres, H. A. 2007. Aves de importancia para la conservación de Tamaulipas. TURevista Digital U@T. http:// www.turevista.uat.edu.mx/; última consulta: 1.V.2009.

Garza-Torres, H. A. y A. G. Navarro. 2003. Breeding records of the sooty tern in Tamaulipas and its distribution in the Gulf of Mexico. Huitzil 4:22-25.

Garza-Torres, H. A., J. R. Herrera-Herrera, G. Escalona-Segura, J. Vargas-Contreras y A. G. Navarro. 2003. New bird records from Tamaulipas, Mexico. Southwestern Naturalist 48:707710.

Gómez, J., Monrós S. J. y J. A. Gil-Delgado. 2000. Notas sobre la biología reproductora de la tángara azuleja Thraupis episcopus, en Santa Clara (Costa Rica). Ardeola 47:265-267.

Haverschmidt, V. F. 1954. Zur Brutbiologie von Thraupis episcopus in Surinam. Journal für Ornithologie 95:48-55.

Howell, G. S. N. y S. Webb. 1995. A guide to the birds of Mexico 
and Northern Central America. Oxford University Press, New York. 851 p.

Ibáñez-Hernández, P. G. y T. Álvarez-Solórzano. 2007. Listados Faunísticos de México. XII. Aves de la Reserva de Ría Lagartos, Yucatán, Instituto de Biología, UNAM, México, D.F. 73 p.

Ingels, J. 1978. The nesting of three tanagers common in French Guiana. Avicultural Magazine 84:105-110.

Instituto Nacional de Ecología. 1997. Guía de aves canoras y de ornato. INE-SEMARNAP/ CONABIO, México, D. F. 177 p.

Isler, M. L. y P. R. Isler. 1987. The tanagers: natural history, distribution and identification. Smithsonian Institution Press, Washington, D. C. 404 p.

Lowery, G. H. Jr. y R. J. Newman. 1951. Notes on the ornithology of southeastern San Luis Potosí. Wilson Bulletin 63:315-322.

MacGregor-Fors, I. 2005. Listado ornitológico del Centro Universitario de Ciencias Biológicas y Agropecuarias, Universidad de Guadalajara, Jalisco. México: un espacio suburbano. Huitzil 6:1-6.

MacGregor-Fors, I. 2008. Relation between habitat attributes and bird richness in western Mexico. Landscape Urban Planning 84:92-98.

MacGregor-Fors, I., J. E. Schondube, L. Morales-Pérez y J. Quesada. 2008. Ampliación del ámbito geográfico-altitudinal y uso de hábitats suburbanos por la mascarita pico grueso Geothlypis poliocephala. Revista Mexicana de Biodiversidad 79:533-534.

Martínez-Morales, M. A., I. Zuria, L. Chapa-Vargas, I. MacGregor-Fors, R. Ortega-Álvarez, E. Romero-Águila y P. Carbó. 2010. Current distribution and predicted geographic expansion of the Rufous-backed Robin in Mexico: a fading endemism? Diversity and Distributions 16:786-797.

Monroy, O. A. y M. Grosselet. 2008. Registros de la tángara azul gris (Tharaupis episcopus) en el estado de Nuevo León. Huitzil 9:29-31.

Monroy, O. A. y N. E. Sánchez Morales. 2009. Registros del mirlo pinto (Ridgwayia pinicola) para el estado de Nuevo León, México. Huitzil 10:19-21.

Navarro, A. G., A. T. Peterson y A. Gordillo-Martínez. 2003. Museums working together: the atlas of the birds of Mexico. In Why museums matter: avian archives in an age of extinction, N. Collar, C. Fisher y C. Feare (eds.). Bulletin British Ornithologists' Club Supplement 123A:207-225 p.

Pablo, L. R. E. 2009. Primer registro del perico argentino (Myiopsitta monachus) en Oaxaca, México. Huitzil 10:4851.

Peterson, A. T., V. Sánchez-Cordero, E. Martínez-Meyer y A. G. Navarro-Sigüenza. 2006. Tracking population extirpations via melding ecological niche modeling with land-cover information. Ecological Modelling 195:229-236.

Peterson, R. T. y E. L. Chalif. 1989. Aves de México. Guía de campo. Diana, México, D. F. 473 p.
Ramírez-Albores, J. E. 2008. Comunidad de aves de la Facultad de Estudios Superiores Zaragoza campus II, UNAM, Ciudad de México. Huitzil. 9:12-19.

Ríos-Muñoz, C. A. y A. G. Navarro-Sigüenza. 2009. Efectos del cambio de uso de suelo en la disponibilidad hipotética de hábitat para los psitácidos de México. Ornitología Neotropical 20:491-509.

Rojas-Soto, O. R. y A. de Alba B. 2002. Record of the Hooded Merganser (Lophodytes cucullatus) in "Los Petenes", northwestern Campeche, Mexico. Ornitología Neotropical 13:85-86

Rzedowski, J. 1978. Vegetación de México. CONABIO, México, D. F. 432 p.

Rzedowski, J. 1990. Vegetación potencial. IV. 8.2. Atlas Nacional de México, vol. II. Escala 1:4, 000,000. Instituto de Geografía, UNAM, México, D. F.

Sánchez-Cordero, V. y E. Martínez-Meyer. 2000. Museum specimen data predict crop damage by tropical rodents. Proceedings of the National Academy of Sciences 97:70747077.

Sánchez-Cordero, V., P. Illoldi-Rangel, M. A. Linaje, S. Sarkar y A. T. Peterson. 2005. Deforestation and extend distributions of Mexican endemic mammals. Biological Conservation 126:465-473.

Sekercioglu, C. H. 2006. Increasing awareness of avian ecological function. Trends in Ecology and Evolution. 21:465-471.

Sekercioglu, C. H., G. C. Daily y P. R. Ehrlich. 2004. Ecosystem consequences of bird declines. Proceedings of the National Academy of Sciences 101:18042-18047.

SEMARNAT (Secretaría de Medio Ambiente, Recursos Naturales y Pesca)/ Instituto de Geografía, UNAM). 2001. Inventario Forestal Nacional 2000-2001. Escala 1:250000. (www.igeograf.unam.mx.semarnt.gob.mx; www.inegi.gob. mx); última consulta: 20.X.2009.

SEMARNAT (Secretaría del Medio Ambiente y Recursos Naturales)/ Instituto de Geografía, UNAM. 2002. Inventario Nacional Forestal. Mapa digitalizado. México, D. F.

Silva, J. M. C. da, C. Uhl y G. Murray. 1996. Plant succession, landscape management, and the ecology of frugivorous birds in abandoned amazonian pastures. Conservation Biology 10:491-503.

Skutch, A. F. 1989. Life of the tanager. Cornell. University Press. New York.

Snow K. B. y D.W. Snow. 1971. The feeding ecology of tanagers and honeycreepers in Trinidad. Auk 88:291-322.

StatSoft. 1998. Statistica for Windows. Computer program manual. Tulsa, Oklahoma.http://www.statsoft.com; última consulta: 10.X.2009.

Trejo, R. I. y R. Dirzo. 2000. Deforestation of seasonally dry tropical forest: a national local analysis in Mexico. Biological Conservation 94:133-142.

Velázquez, A., J. F. Mas, J. R. Díaz-Gallegos, R. Mayorga- 
Saucedo, P. C. Alcántara, R. Castro, T. Fernández, G. Bocco, E. Escurra y J. L. Palacios. 2002. Patrones y tasas de cambio de uso de suelo en México. Gaceta Ecológica, nueva época
62:21-38

Zar, H. J. 1999. Biostatistical analysis, cuarta edición. PrenticeHall, New Jersey. 718 p. 